\title{
Determination of amylopectin structure and physicochemical properties in rice endosperm starch of mutant lines derived from Malaysian rice cultivar MR219
}

\begin{abstract}
BACKGROUND: Characterization of starch properties and functionality can apply breeding program selection for desirable traits such as eating, cooking and processing qualities to meet consumer preference. Low amylose content is generally preferred in Malaysia because of cohesive, tender and glossy cooked rice. Rice high in short-chain amylopectin has a lower transition temperature of starch gelatinization. In the continuing search for improved starch quality in rice cultivars a study was carried out with new mutant lines MR219-4 and MR2199, derived from MR219. RESULTS: MR219 and its mutant lines contain L-type amylopectin, being high in amylopectin of intermediate chain length with degree of polymerization 12-21. The apparent amylose content (AAC) in MR219-4 had lower AAC value (19.2\%) compared to other lines. A strongly negative correlation was found between chain-length fraction of amylopectin and transition temperatures $ठ$ onset temperature peak temperature, and conclusion temperature $(0.992,0.958,0.950 ; \mathrm{P}<0.01) \circlearrowright$ with fraction b1 (fb1), respectively. CONCLUSION: The Malaysian lines studied contain L-type amylopectin and offer a better understanding of grain quality improvement in terms of starch properties and functionality. This information will be directly applicable to select for desirable traits in future breeding programs.
\end{abstract}

Keyword: Amylopectin; Apparent amylose content (AAC); MR lines; Thermal properties; Pasting properties; Rice (Oryza sativa L.) 